\title{
The expression and phosphorylation of ezrin and merlin in human pancreatic cancer
}

\author{
JIAHUA ZHOU ${ }^{1}$, YONGJIANG FENG ${ }^{1}$, KETAO TAO $^{1}$, ZHANHAI SU ${ }^{2}$, \\ XIAOJIN YU ${ }^{3}$, JIE ZHENG $^{4}$, LIHUA ZHANG $^{5}$ and DETONG YANG ${ }^{1}$

\begin{abstract}
${ }^{1}$ Department of General Surgery, Zhongda Hospital, School of Medicine, Southeast University, Nanjing 210009;
${ }^{2}$ Basic Medical Research Center, Qinghai University, Xining 810001; ${ }^{3}$ Department of Epidemiology and Biostatistics,

School of Public Health, Southeast University; ${ }^{4}$ Department of Pathology, School of Medicine, Southeast University;

${ }^{5}$ Department of Surgical Pathology, Zhongda Hospital, School of Medicine, Southeast University, Nanjing 210009, P.R. China
\end{abstract}

Received February 1, 2014; Accepted March 21, 2014

DOI: $10.3892 /$ ijo.2014.2381

\begin{abstract}
Pancreatic carcinoma is the most common pancreatic malignancy and is associated with a very poor prognosis. Therefore, new prognostic factors and new treatment strategies are clearly needed. In this study, we retrospectively studied the levels of phosphorylated ezrin in 19 patients with pancreatic carcinoma by immunohistochemical analysis and determined the correlation between protein expression, clinicopathological characteristics and prognosis in pancreatic adenocarcinoma. We also characterized the phenotype of the overexpression of wild-type and phosphorylated ezrin and merlin in human pancreatic cancer cell lines. A significant correlation between the levels of phosphorylated ezrin 353 and ezrin 567 and the stage of pancreatic cancer was observed. Moreover, Kaplan-Meier analysis revealed that patients with high levels of phosphorylated ezrin had a significantly poorer survival rate $(\mathrm{P}<0.05)$. In addition, the overexpression of wildtype merlin or ezrin inhibited cell proliferation, migration and adhesion. However, the overexpression of T567D ezrin, a mutant that mimics permanent phosphorylation, promoted the proliferation, adhesion and migration of the pancreatic adenocarcinoma cell line SW1990. The overexpression of S518D merlin inhibited the growth of SW1990 and did not affect migration or adhesion. These results suggest that the phosphorylation of ezrin may contribute to the progression
\end{abstract}

Correspondence to: Dr Jiahua Zhou, Department of General Surgery, Zhongda Hospital, School of Medicine, Southeast University, 87 Dingjiaqiao Road, Nanjing 210009, P.R. China

E-mail: zhoujh@seu.edu.cn

Abbreviations: UICC, the International Union Against Cancer; TNM, Classification of Malignant Tumors; DAB, 3,3'-diaminobenzidine; MTT, 3-(4,5-dimethylthiazol-2-yl)-2,5-diphenyltetrazolium bromide

Key words: pancreatic cancer, ezrin, merlin, phosphorylation, prognosis of pancreatic carcinoma and that the level of phosphorylated ezrin may serve as an adverse prognostic factor for pancreatic carcinoma.

\section{Introduction}

Pancreatic cancer is a common type of tumor, and its incidence has increased in recent years (1-3). The effectiveness of pancreatic cancer therapy is unclear, and $<3 \%$ of patients live for more than five years. As a result, the morbidity and mortality rates for pancreatic cancer are very similar. The main factors that affect the prognosis of pancreatic cancer are the difficulty of early diagnosis, the low rate of radical surgical resection, and the insensitivity to chemotherapy and radiotherapy. Pancreatic carcinogenesis and metastasis are believed to be correlated with oncogene activation, the inactivation of tumor suppressor genes, and the aberrant regulation of molecular signaling pathways, such as those used by the ERM family (4).

Ezrin and merlin display $45 \%$ nucleotide sequence identity and play important roles in the linkage between transmembrane proteins and the cytoskeleton. Both proteins belong to the band 4.1 protein superfamily (5). The members of the ezrin/radixin/moesin (ERM) family display $75-80 \%$ sequence identity in humans (6). Ezrin proteins are localized at regions related to cytoskeletal remodeling, such as ruffling membranes, dynamic actin filaments, and the cleavage furrows of dividing cells (7). Active ezrin protein plays critical roles in connecting actin filaments to the membrane. Ezrin protein expression is closely associated with cell differentiation, adhesion, metastasis, invasion, and the extent of cancer malignancy (8-10). Ezrin mutations and abnormal protein expression have been observed in many types of cancer. Merlin is encoded by the tumor-suppressing gene NF2. Merlin proteins are found in the same intracellular locations as ezrins. Merlin proteins can directly or indirectly interact with many other types of proteins. The merlin protein plays a key role in the contact inhibition of cell growth and in signal transduction. When the NF2 gene is mutated, the merlin protein becomes inactive and loses its tumor-suppressing function, resulting in tumorigenesis (11). 
Ezrin and merlin protein molecules consist of three basic functional regions: a spherical membrane-bound N-terminal domain, an $\alpha$-helical domain, and a positively charged C-terminal domain (12). The binding sites between the plasma membrane and actin are hidden because the $\mathrm{N}$ - and C-termini of the ERM proteins are molecularly bound under normal conditions. In response to certain types of stimulation, ERM proteins are phosphorylated by a protein kinase, thereby causing the ERM protein to be uncovered. The phosphorylated N-terminus of the ERM protein can then interact with CD44, CD43 and L-selectin, enabling the transfer of information related to the cell membrane protein status. The C-terminus can connect to the F-actin protein, which mediates the reorganization of muscle actin and cytoskeletal activities within the cell. Such mediation may be related to tumorigenesis and metastasis.

Merlin has two subtypes, merlin-1 and merlin-2. Merlin-1 exists in both closed and open conformations, and it is these versions that undergo phosphorylation (13). Closed merlin-1 inhibits tumor growth, but open merlin-1 does not. Merlin-2, which lacks exon 17, has only an open form and does not inhibit tumor growth (14). The present study demonstrates that merlin is phosphorylated at Ser518. The p21 activation kinase (PAKs) and cAMP-dependent protein kinase A regulate the phosphorylation of merlin $(15,16)$. Phosphorylated merlin can easily form heterodimers with ezrin proteins, which changes the status of the merlin protein from a growth-inhibitory state to an open state. Jin et al described two proteins that interact with merlin (17), the myosin phosphatase MYPT1 PP1- $\delta$ and CPI-17 (protein kinase C-potentiated phosphatase inhibitor of $17 \mathrm{kDa}$ ). MYPT1 PP1- $\delta$ is involved in the dephosphorylation of merlin protein, thereby activating the protein and suppressing tumor growth. CPI-17 is a MYPT1 PP1- $\delta$ inhibitor that inactivates merlin and promotes tumor growth. However, the specific role(s) of phosphorylated merlin are unclear.

A previous analysis of more than 5,000 samples of tumor and normal tissues (18) revealed that nearly all tumor and normal tissues exhibit different degrees of ezrin expression. Another study indicated high levels of ezrin expression and activation in many metastatic tumor cells (19). Yu et al observed that ezrin is involved in the metastasis of malignant tumors from different tissues (20). Ohtani et al showed that the ability of endometrial carcinoma to invade and metastasize declines after ezrin levels decrease (21). However, another study showed that invasion and metastasis can increase significantly in colorectal cancer cells when ezrin expression is inhibited (22). In 2009, Ren et al observed changes in the dynamic regulation of phosphorylated ERM proteins during different periods of the formation and metastasis of osteosarcomas (23).

The purpose of the present study was to evaluate the expression of ERM and merlin in human pancreatic cancer tissues and cell lines and to determine the relationships of these proteins with tumorigenesis and metastasis.

\section{Materials and methods}

Cell culture and tumor samples. The human pancreatic cancer cell line SW1990 was cultured in vitro in RPMI-1640
(Gibco BRL, China) with $10 \%$ inactivated fetal bovine serum, $100 \mathrm{U} / \mathrm{ml}$ penicillin, and $100 \mathrm{mg} / \mathrm{ml}$ streptomycin (Gibco $\mathrm{BRL}$ ) under a $5 \% \mathrm{CO}_{2}$ atmosphere at $37^{\circ} \mathrm{C}$. The $\mathrm{SW} 1990$ cells were obtained from the cell bank at the Chinese Academy of Sciences. A total of 19 paraffin-embedded tissue specimens of pancreatic cancer were obtained from patients undergoing surgical resection from June 2006 to September 2008 at Southeast University Affiliated Zhongda Hospital. Pancreatic cancer was histologically confirmed in patients who did not undergo anti-tumor therapy before their operations. All patients completed postoperative follow-up. The survival time spanned the period from the date of surgery to the date of death due to recurrence or metastasis. The 2010 version of the International Union Against Cancer (UICC) standards was used for the Classification of Malignant Tumors (TNM) staging (24). The ethics committee of Southeast University Affiliated Zhongda Hospital reviewed and approved this study. The participants provided their written consent to participate in this study.

Immunohistochemistry. Paraffin-embedded sections from surgical specimens were deparaffinized, rehydrated and immersed in $3 \%$ hydrogen peroxide, incubated for $30 \mathrm{~min}$ at room temperature, and washed 3 times with PBS. Antigen retrieval was performed by heating the slides in $0.01 \mathrm{~mol} / 1$ sodium citrate buffer ( $\mathrm{pH} \mathrm{6.0)}$ for $30 \mathrm{~min}$ at $95^{\circ} \mathrm{C}$. The cooled slides were then incubated in $10 \%$ normal blocking goat serum for $30 \mathrm{~min}$, after which they were then incubated with an antibody concentration of 1:200 overnight at $4^{\circ} \mathrm{C}$. The next day, the slides were warmed to room temperature and processed using the labeled horseradish-peroxidase method at $37^{\circ} \mathrm{C}$ for $15 \mathrm{~min}$. The slides were washed three times for 5 min each in PBS and stained with 3,3'-diaminobenzidine (DAB). A total of 10 fields of view on each slide were analyzed under high magnification (x400, Leitz microscope, Germany) by two pathologists who were double-blinded to the goals of the experiment. According to the intensity of immunostaining, the protein expression was graded as 0 (no staining), 1 (weak staining), 2 (moderate staining), or 3 (strong staining). Cell staining was graded as 0 (no cells stained), 1 ( $<33 \%$ of the cells stained), 2 (34-67\% of the cells stained), or 3 ( $>67 \%$ of the cells stained) based on the proportion of stained tumor cells to all tumor cells in tissue sections. The mathematical products of the staining intensity scores and the staining proportion scores served as total assessment scores. The immunohistochemical analysis was classified as positive (a score of $\geq 3$ ) or negative (a score of $<3$ score) (25).

Plasmid transfection and target gene expression. The recombinant plasmids pcDNA3.1+HA, pcDNA3.1+HA-ezrin, pcDNA3.1+HA-ezrin Thr567 (T567D ezrin, a mutant which mimics permanent phosphorylation), pcDNA3.1+HA-merlin, and pcDNA3.1+HA-merlin Ser518 (S518D merlin) were transfected into SW1990 cells using Lipofectamine 2000. At $24 \mathrm{~h}$ after transfection, the cells were transferred to new sixwell plates at a 1:2 ratio. The transfected cells were cultured in RPMI-1640 containing G418 $(500 \mu \mathrm{g} / \mathrm{ml})$ for another $48 \mathrm{~h}$ until approximately day 12 , when all cells in the control group died. Some of the cells containing the target genome were still visibly alive and exhibited a nested distribution. Monoclonal cells were selected and cultured with G418 at $100 \mu \mathrm{g} / \mathrm{ml}$. 


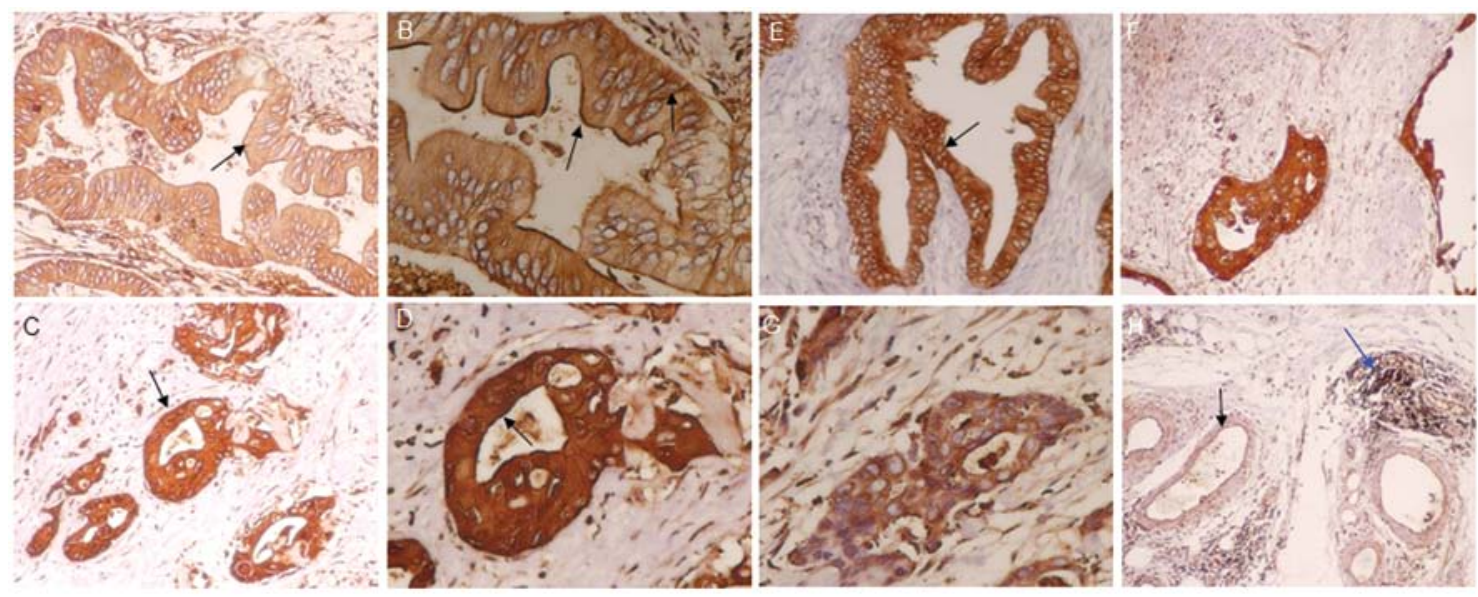

Figure 1. Ezrin and merlin protein expression in normal human pancreas and pancreatic carcinoma samples. (A and B) Positively stained ezrin in the cytoplasm and cell membrane of human pancreatic cancer tissues (A, x200; B, x400). (C and D) Representative p-ezrin 567 positively stained in the cytoplasm and cell membrane of human pancreatic cancer tissues (C, x200; D, x400; black arrows indicate membrane staining). (E) Expression of the p-ezrin 353 protein in human pancreatic cancer tissues (x200, black arrows indicate membrane staining). (F) Expression of the merlin protein in human pancreatic cancer tissues (x200). (G) Expression of the p-merlin 518 protein in human pancreatic cancer tissues (x400). $(\mathrm{H})$ Expression of the ezrin protein in normal pancreatic tissue and blood vessels in the pancreas (x100, black arrows indicate blood vessels; blue arrows indicate islet cells).

Table I. Relationship between TNM stage and protein expression of p-ezrin 353 and p-ezrin 567 in pancreatic cancers.

\begin{tabular}{|c|c|c|c|c|}
\hline \multirow[b]{2}{*}{ TNM stage } & \multicolumn{2}{|c|}{ p-ezrin 353} & \multicolumn{2}{|c|}{ p-ezrin 567} \\
\hline & Expression & No expression & Expression & No expression \\
\hline I & 2 & 2 & 1 & 3 \\
\hline II & 2 & 2 & 2 & 2 \\
\hline III & 6 & 0 & 6 & 0 \\
\hline IV & 5 & $0^{\mathrm{a}}$ & 5 & $0^{\mathrm{b}}$ \\
\hline
\end{tabular}

${ }^{\mathrm{a}} \mathrm{P}=0.046,{ }^{\mathrm{b}} \mathrm{P}=0.011$

Western blot analysis. The cancerous human pancreatic cell lines were either left untreated or treated and washed with cold phosphate-buffered saline (PBS). The four groups of cells were lysed in Laemmli lysis buffer. Equal amounts of lysate were separated by electrophoresis on a $12 \%$ SDS-PAGE gel, and the proteins were transferred to nitrocellulose membranes. The membranes were then blocked with TBS containing 5\% low-fat milk and $0.05 \%$ Tween for $1 \mathrm{~h}$, washed three times with TBS containing $0.05 \%$ Tween, and incubated with specific primary antibodies for $2 \mathrm{~h}$ at room temperature. The membranes were washed with TBS containing $0.05 \%$ Tween and then incubated with peroxidase-conjugated secondary antibodies labeled with horseradish peroxidase (HRP). The images were then developed on X-ray film. The optical density of the bands was calculated using Quantity One software (Bio-Rad Laboratories, Inc.). Mouse anti-human monoclonal anti-ezrin and anti- $\beta$-actin and mouse anti-human polyclonal anti-p-ezrin Thr567 were purchased from Santa Cruz Biotechnology, Inc. (USA) at a dilution of 1:500. Mouse anti-human monoclonal anti-merlin and anti-HA and mouse anti-human polyclonal anti-p-merlin Ser518 were purchased from Cell Signaling Technology (USA) and used at a dilution of $1: 1000$.
Cell proliferation assay. Cell viability was assessed using the MTT assay. Human pancreatic cancer cells (SW1990, SW1990-HA, SW1990-ezrin, SW1990-p-ezrin, SW1990merlin, and SW1990-p-merlin) were cultured in 96-well plates at a density of $1 \times 10^{4}$ cells $/ \mathrm{ml} \mathrm{A} 20-\mu 1$ aliquot of MTT (Amresco) labeling reagent was added to each well containing cells in $150 \mu \mathrm{l}$ medium, and the cells were incubated in a humidified incubator at $37^{\circ} \mathrm{C}$ to permit MTT metabolism for $4 \mathrm{~h}$. The medium was removed, and the cells were re-suspended in formazan in $150 \mu \mathrm{l}$ DMSO. The absorbance of the samples was measured at $490 \mathrm{~nm}$ using a spectrophotometer and microplate reader, and the viability values were expressed as percentages of the controls.

Cell cycle and cell apoptosis. Cells in logarithmic growth phase (SW1990, SW1990-HA, SW1990-p-ezrin, and SW1990-p-merlin) were harvested at an adjusted cell concentration of $1 \times 10^{6} / \mathrm{ml}$. The prepared single-cell suspension was fixed in pre-cooled $70 \%$ ethanol. The fixative was then washed away with PBS, the samples were stained with propidium iodide (Sigma), and RNase A was added. The cells were then used to detect the cell cycle and apoptosis using flow cytometry. 

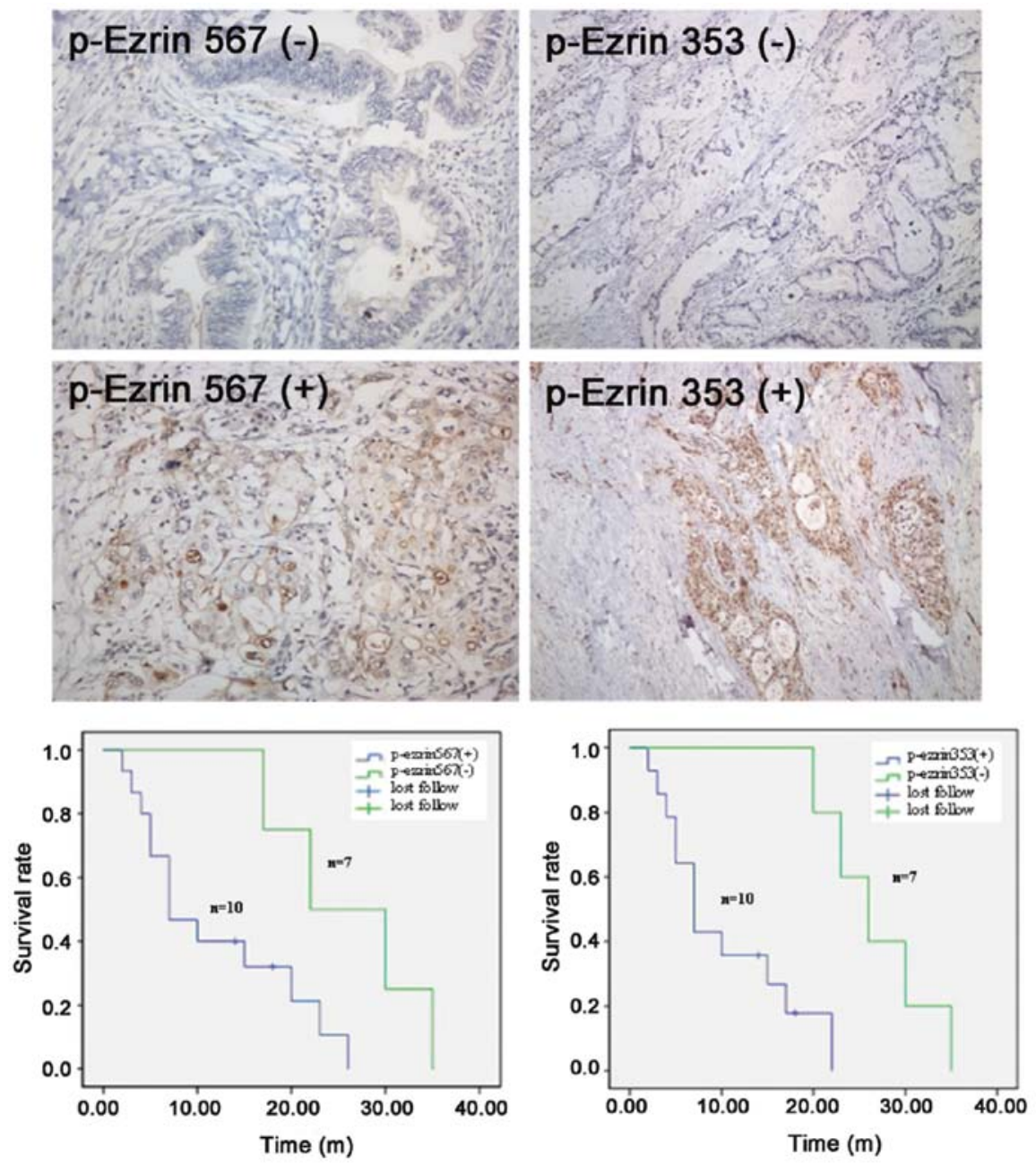

Figure 2. Survival curves of pancreatic cancer patients in the p-ezrin 567- and p-ezrin 353-positive and -negative expression groups.

Transwell migration assay. Logarithmic growth-phase cells (SW1990, SW1990-HA, SW1990-p-ezrin and SW1990-pmerlin) were harvested, digested, and counted. The cells were placed in the upper chamber of a Transwell containing $200 \mu \mathrm{l}$ serum-free medium containing $0.1 \%$ BSA at a density of $1 \times 10^{5} / \mathrm{ml}$. The lower chamber of the Transwell contained $500 \mu \mathrm{l}$ medium containing $10 \%$ fetal bovine serum in $24-w e l l$ plates at room temperature. After an incubation period of $12 \mathrm{~h}$, the cells that had migrated through the membrane were stained with H\&E and counted.

Cell-matrix adhesion assay. The 96-well plates were coated with Matrigel and incubated overnight at $4^{\circ} \mathrm{C}$. To each well was added $50 \mu \mathrm{l}$ serum-free medium containing $0.1 \% \mathrm{BSA}$, and the plates were incubated at $37^{\circ} \mathrm{C}$ for $30 \mathrm{~min}$. The cells in the $200-\mu 1$ cell suspension were seeded at a final concentration of $1 \times 10^{5} / \mathrm{ml}$ and incubated at $37^{\circ} \mathrm{C}$ for 1 or $2 \mathrm{~h}$ so that each group consisted of 4 parallel samples. The cell absorbance of each well was determined using the MTT assay with a reference to the control (the basement membrane of the BSA group).

Statistical analysis. The results are expressed as the means \pm standard deviations (SD). Data analyses were performed using SPSS 17.0. Fisher exact tests were used for comparisons between 2 sample proportions, and the Pearson
Chi-squared test was used for comparisons of $>2$ sample proportions. The Kaplan-Meier analysis and log-rank test were employed for survival analysis. P-values $<0.05$ and $<0.01$ were considered statistically significant.

\section{Results}

Protein expression of ezrin and merlin in normal human pancreas and pancreatic carcinoma tissues. Ezrin Thr567, ezrin Tyr353, merlin Ser518, p-ezrin Thr567, p-ezrin Tyr353 and p-merlin Ser518 showed different levels of protein expression in tissue samples from normal human pancreases and pancreatic carcinoma (Fig. 1). The expression of the 6 target proteins did not significantly differ among these samples. These proteins were seldom present in the cell membranes of normal pancreatic cells. The expression of 4 proteins (ezrin Thr567, p-ezrin Thr567, ezrin Tyr353, p-ezrin Tyr353) was 94.7, $63.2,94.7$, and $73.7 \%$ higher, respectively, in the membranes of pancreatic cancer cells than in normal cell membranes $(\mathrm{P}<0.05)$. The expression of merlin Ser518 and p-merlin Ser518 protein was lower in the cell membranes of pancreatic cancer samples than in normal cell membranes.

The relationship between clinicopathological factors and protein expression of ezrin and merlin in pancreatic cancer. 
A

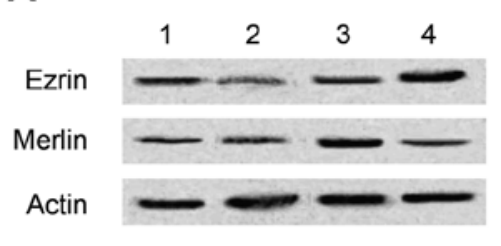

C

D

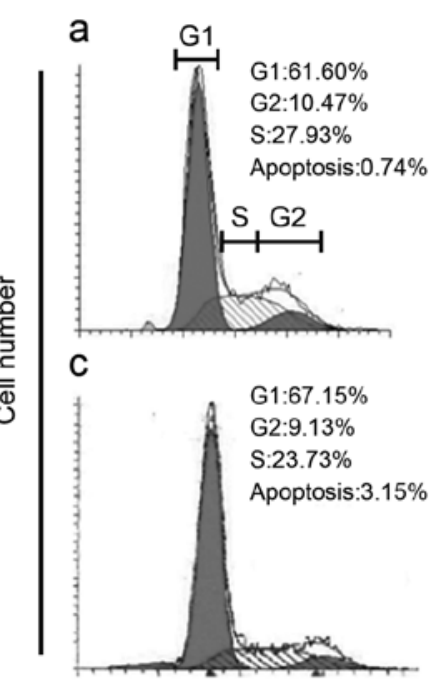

b

B
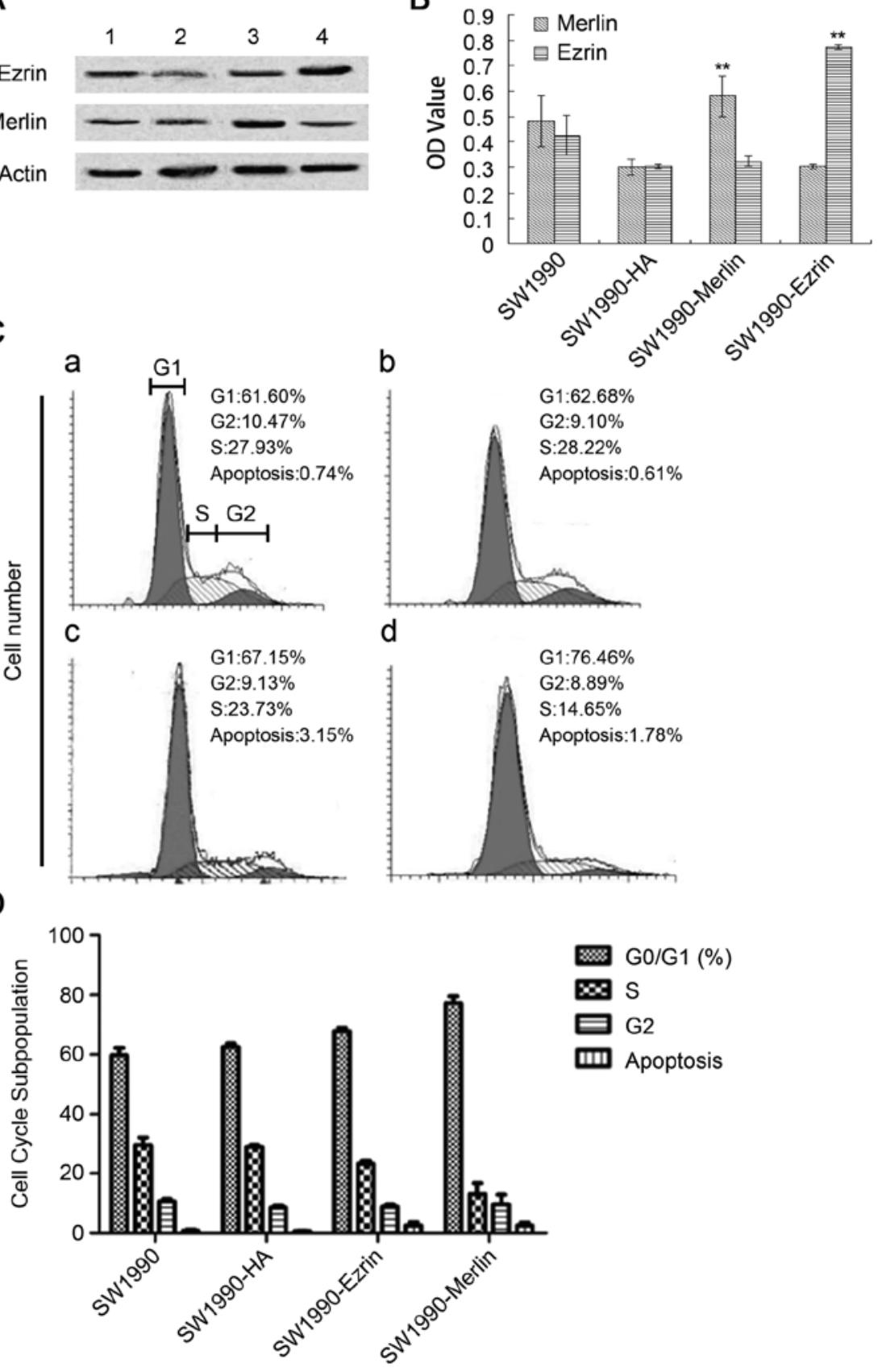

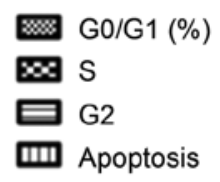

Figure 3. Effects of the overexpression of wild-type ezrin and merlin on proliferation and cell cycle distribution in SW1990 cells. (A and B) Wild-type ezrin and merlin were stably overexpressed in SW1990 cells after transfection with the wild-type ezrin and merlin genes. (C and D) Cell cycle distribution of SW1990 cells overexpressing wild-type ezrin and merlin.

The level of expression of p-ezrin 353 and p-ezrin 567 in the cytoplasm of pancreatic cancer cells increased with TNM stage $(\mathrm{P}<0.05)$. The levels of expression were not associated with gender, age, tumor location or level of differentiation, and were also independent of lymph node metastasis or neural invasion. In addition, the clinicopathological factors did not correlate with the expression of any of the 4 other proteins, ezrin Thr567, ezrin Tyr353, merlin Ser518, or p-merlin Ser518 (Table I).

The relationship between p-ezrin expression and the survival period after pancreatic cancer treatment. Of a total of 19 cases, 17 were followed up, and 2 were lost to follow-up. The follow-up time ranged from 2 to 35 months. The survival time for the group positive for p-ezrin 567 expression in the cytoplasm of pancreatic cancer cells was 2-26 months, and the average survival time was 11.9 months. For patients negative for p-ezrin 567 expression, the survival time was 17-35 months, and the median survival time was 26.0 months. Using a logrank test, the difference between these two groups was found to be statistically significant $\left(\chi^{2}=4.211, \mathrm{P}=0.040\right)$. The survival of the group of patients positive for p-ezrin 353 expression was similar to those positive for p-ezrin 567; the survival time was 2-22 months, and the median survival time was 10.4 months. Individuals negative for p-exrin 353 expression exhibited a survival time of 20-35 months and a median survival time of 
A

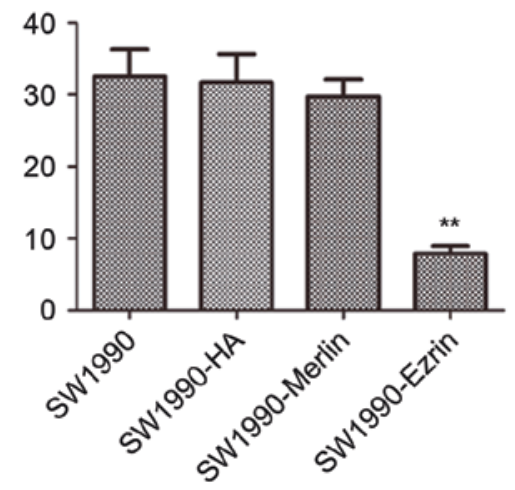

B

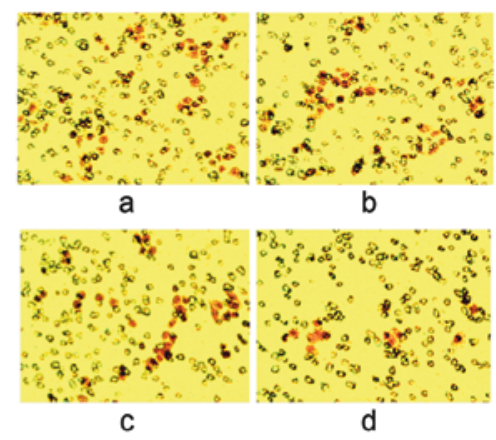

C

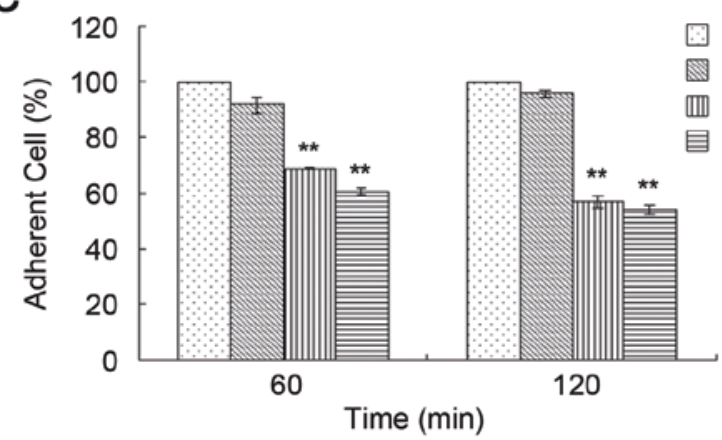

SW1990

SW1990-HA

SW1990-Ezrin

SW1990-Merlin

Time $(\min )$

Figure 4. Effects of the overexpression of wild-type ezrin and merlin on the migration and matrix adhesion ability of SW1990 cells. (A and B) Migration, (C) Matrix adhesion ability.

26.8 months. Using Kaplan-Meier analysis and the log-rank test, this difference was found to be statistically significant $\left(\chi^{2}=8.813, \mathrm{P}=0.003\right)$ (Fig. 2).

Effect of the overexpression of wild-type ezrin and merlin on SW1990. Our results demonstrated that the plasmid was successfully transfected into cells and was stably expressed. The proteins ezrin and merlin were highly expressed (Fig. 3A and B). Overexpression of wild-type ezrin and merlin inhibited the proliferation and growth of pancreatic cancer cells. The MTT results demonstrated that the cellular growth rate and proliferative capacity were significantly lower in the plasmidtransfection group (SW1990-ezrin and SW1990-merlin) than in untransfected cells (SW1990 cell line) or in cells transfected with a blank plasmid (SW1990-HA) $(\mathrm{P}<0.01)$.

Flow cytometric analysis revealed a significant increase in the fraction of cells in G0/G1 phase and a significant decrease in the fraction of cells in S phase $(\mathrm{P}<0.05)$ for SW1990ezrin and SW1990-merlin cells compared to SW1990 and SW1990-HA cells. More SW199-merlin cells than SW1990ezrin cells were in G0/G1 phase (77.29 $\pm 2.27 \%)$; conversely, more SW1990-ezrin cells than SW1990-merlin cells were in $\mathrm{S}$ phase $(\mathrm{P}<0.01)$. The difference in the rates of apoptosis was not statistically significant between the two transfected cell types $(\mathrm{P}>0.05)$. The results demonstrated that the effect of merlin on the cell cycle was more pronounced than that of ezrin. Furthermore, the overexpression of ezrin and merlin did not significantly affect the apoptosis of SW1990 (Fig. 3C and D).

The number of SW1990-HA cells, SW1990-ezrin cells, and untransfected SW1990 cells that traversed the basement membrane did not significantly differ as assessed by a Transwell test $(\mathrm{P}>0.05)$. The number of SW1990-merlin cells that passed through the basement membrane was significantly lower than the number of SW1990 cells $(\mathrm{P}<0.05)$. However, the matrix adhesion ability of SW1990-ezrin and SW1990-merlin cells was significantly decreased at $60 \mathrm{~min}$ and $120 \mathrm{~min}$ compared with untransfected cells and SW1990-HA cells $(\mathrm{P}<0.01)$ (Fig. 4).

Effect of the overexpression of phosphorylated ezrin and merlin on SW1990. Our results demonstrated that the plasmid (a mutant which mimics permanent phosphorylation) was successfully transfected into cells and was stably expressed. The phosphorylated ezrin and merlin proteins were highly expressed (Fig. 5A and B).

Overexpression of T567D ezrin (p-ezrin), a mutant that mimics permanent phosphorylation, in SW1990-p-ezrin cells promoted proliferation, and both the proliferative capacity and growth rate of SW1990-p-merlin cells decreased significantly upon overexpression of S518D merlin $(\mathrm{p}-$ merlin) $(\mathrm{P}<0.05)$ (Fig. 5C).

Fewer cells were in G0/G1 phase in SW1990-p-ezrin cells than in SW1990 cells, and more cells were in S phase and G2 phase. Both proliferation and the rate of apoptosis were increased in SW1990-p-ezrin cells compared to SW1990 cells $(\mathrm{P}<0.05)$. The SW1990-p-merlin cells ceased in G1 phase, and the rate of apoptosis also decreased $(\mathrm{P}<0.05)$ (Fig. 5D and E).

More SW1990-p-ezrin cells passed through the basement membrane than SW1990-HA and SW1990 cells $(\mathrm{P}<0.05)$. This difference suggests that ezrin protein phosphorylation 

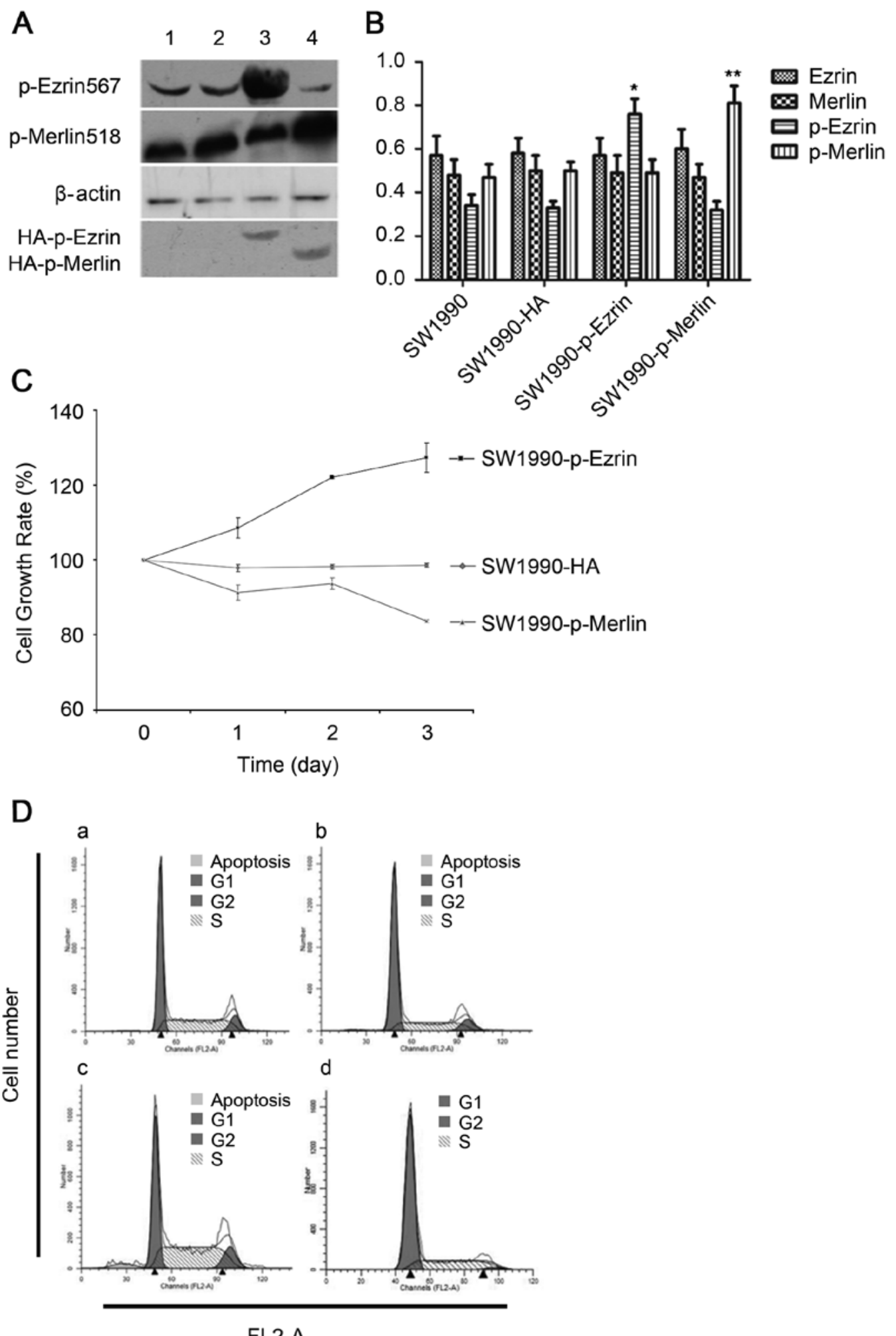

Figure 5. Effects of the overexpression of phosphorylated ezrin and merlin on the proliferation and cell cycle distribution of SW1990 cells. (A and B) Phosphorylated ezrin and merlin were stably overexpressed in SW1990 cells after transfection with the genes encoding phosphorylated ezrin and merlin. (C) Growth curves of cells after transfection with p-ezrin and p-merlin. (D, a-d) Cell cycle distribution of SW1990 cells overexpressing p-ezrin and p-merlin.

promotes the migration of SW1990 cells. There were no significant differences in migration among SW1990 cells, SW1990-HA cells, and SW1990-p-merlin cells ( $>$ >0.05). (Fig. 6A and B) Although overexpressing wild-type merlin has been shown to inhibit the migration of SW1990 cells, overexpressing phosphorylated merlin does not.
The ability of SW1990-p-ezrin cells to adhere to the matrix significantly increased at $60 \mathrm{~min}$ and $120 \mathrm{~min}$ $(\mathrm{P}<0.05)$ (Fig. 6C). The matrix adhesion ability of SW1990p-merlin was not significantly different from that of SW1990 and SW1990-HA cells $(\mathrm{P}>0.05)$. These results demonstrate that ezrin phosphorylation enhances cell adhesion and that 


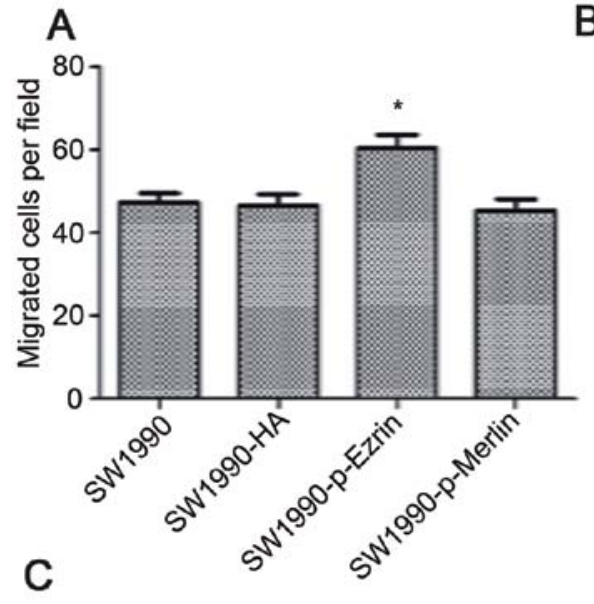

B
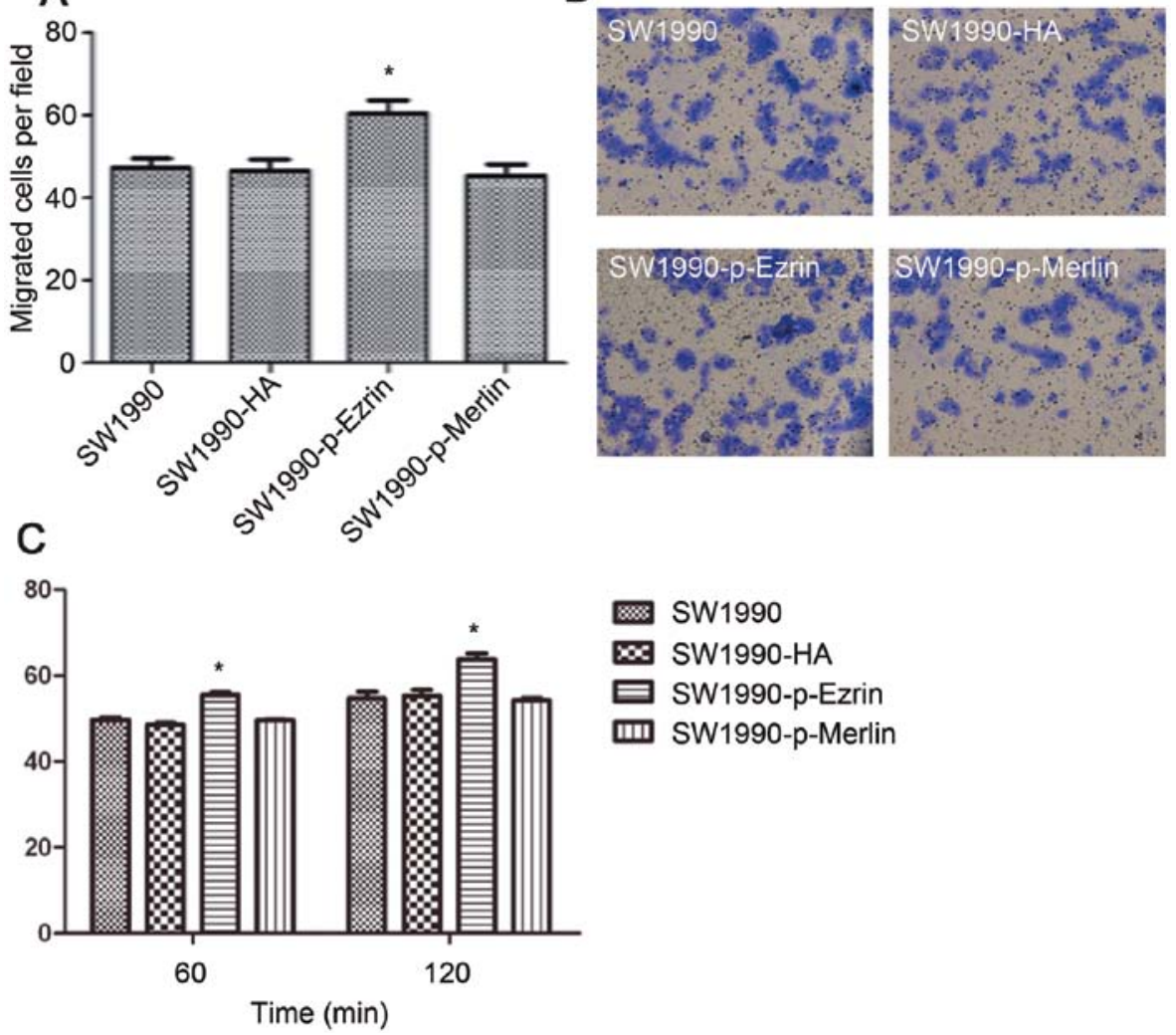

Figure 6. Effects of the overexpression of phosphorylated ezrin and merlin on the migration and matrix adhesion ability of SW1990 cells. (A and B) Migration, (C) Matrix adhesion ability.

the wild-type merlin protein does not inhibit the adhesion of SW1990 cells. Adhesion appears to be lost after phosphorylation.

\section{Discussion}

Our results showed that the levels of p-ezrin 567/p-ezrin 353 protein expression in the cytoplasm increased with TNM stage of human pancreatic cancers. The survival time of the group positive for p-ezrin 567/p-ezrin 353 protein expression was shorter than that of the negative group. Moreover, we demonstrated that overexpression of T567D ezrin, a mutant that mimics permanent phosphorylation, promotes the proliferation, adhesion, and migration of pancreatic adenocarcinoma in vitro.

Cui et al performed immunohistochemical analysis of the postoperative specimens of 66 patients with pancreatic cancer (25), which revealed that positive expression of p-ezrin Thr353 was associated with poor cell differentiation and an increased likelihood of lymph node metastasis in pancreatic cancer. The survival time of pancreatic cancer patients positive for $\mathrm{p}$-ezrin 353 expression differed from those negative for pezrin 353 expression. The difference in the survival time between the groups positive for p-ezrin 567/pezrin 353 protein expression and those negative for expression was statistically significant. Coupled with our observation that the rates of p-ezrin 567/p-ezrin 353 protein expression in the cytoplasm increase with TNM stage of pancreatic cancers, these results suggest a close relationship between the phosphorylation status of ezrin and the adverse biological behaviors of human pancreatic cancer, such as the capacity for cell growth, invasion and metastasis.

Ren et al (23) reported that the phosphorylation of the ERM protein caused dynamic changes in the regulation of different periods during the onset of metastasis in osteosarcomas. They also demonstrated that the ezrin protein was widely distributed in tumor tissues and that the concentration of the phosphorylated ERM protein was higher at the margins of the invading tumor. We did not observe this phenomenon.

Hunter (19) observed high levels of ezrin expression and ezrin protein activation in many metastatic tumor cell types, such as osteosarcomas and rhabdomyosarcomas. The ezrin protein is known to be involved in the metastasis of malignant tumors from various tissues (20). By contrast, Hiscox and Jiang (22) demonstrated that tumor cell invasion and metastasis increased significantly in colorectal cancer cells when the level of ezrin expression was suppressed. Similarly, poor prognosis in ovarian cancer is closely related to low ezrin expression and ezrin deletions $(26,27)$. In the present study, we did not observe an obvious change in the migration of SW1990 cells after transfection with ezrin. However, growth, adhesion, migration and invasion increased markedly after transfection with phosphorylated ezrin. This finding suggests that the ezrin protein affects pancreatic cancer via phosphorylation.

The capacity for growth, proliferation, migration and cell matrix adhesion was significantly lower in SW1990-merlin cells than in the control cells, which indicates that merlin 
inhibits tumor cell metastasis as well as tumor growth. Transfection of SW1990 cells with p-merlin resulted in a decrease in cell proliferation compared to control cells, but cell adhesion, migration, and invasion did not change.

Our study demonstrates that the expression of phosphorylated ezrin proteins is related to the clinical and pathological features of pancreatic cancer. P-ezrin plays a positive regulatory role in the growth, adhesion, and invasion of SW1990 cells, while p-merlin serves a negative regulatory role.

In conclusion, the phosphorylation of ezrin may contribute to the progression of pancreatic carcinoma, and the level of phosphorylated ezrin may serve as an adverse prognostic factor for pancreatic carcinoma.

\section{Acknowledgements}

This study was supported by the China National Natural Science Foundation (no. 81071967 and 30872500).

\section{References}

1. Jemal A, Bray F, Center MM, et al: Global cancer statistics. CA Cancer J Clin 61: 69-90, 2011

2. Chen WQ, Wang QS, Zhang SW, et al: An analysis of incidence and mortality of pancreas cancer in China 2003-2007. China Cancer 21: 248-253, 2012.

3. Siegel R, Naishadham D and Jemal A: Cancer Statistics, 2012. CA Cancer J Clin 62: 10-29, 2012.

4. Cartwright T, Richards DA and Boehm KA: Cancer of the pancreas: are we making progress? A review of studies in the US Oncology Research Network. Cancer Control 15: 308-313, 2008

5. Wan X, Mendoza A, Khanna C and Helman LJ: Rapamycin inhibits ezrin-mediated metastatic behavior in a murine model of osteosarcoma. Cancer Res 65: 2406-2411,2005.

6. Diakowski W, Grzybek M and Sikorski AF: Protein 4.1, a component of the erythrocyte membrane skeleton and its related homologue proteins forming the protein 4.1/FERM superfamily Folia Histochem Cytobiol 44: 231-248, 2006.

7. Wakayama T, Nakata H, Kurobo M, et al: Expression, localization, and binding activity of the ezrin/radixin/moesin proteins in the mouse testis. J Histochem Cytochem 57: 351-362, 2009.

8. Arpin M, Chirivino D, Naba A and Zwaenepoel I: Emerging role for ERM proteins in cell adhesion and migration. Cell Adh Migr 5: 199-206, 2011.

9. Jörgren F, Nilbert M, Rambech E, et al: Ezrin expression in rectal cancer predicts time to development of local recurrence. Int J Colorectal Dis 27: 893-899, 2012.
10. Arslan AA, Silvera D, Arju R, et al: Atypical ezrin localization as a marker of locally advanced breast cancer. Breast Cancer Res Treat 134: 981-988, 2012.

11. Evans DG, Ramsden RT, Shenton A, et al: What are the implications in individuals with unilateral vestibular schwannoma and other neurogenic tumors? J Neurosurg 108: 92-96, 2008.

12. Tsukita S, Yonemura S and Tsukita S: ERM proteins: head-totail regulation of actin-plasma membrane interaction. Trends Biochem Sci 22: 53-58, 1997.

13. Ye K: Phosphorylation of merlin regulates its stability and tumor suppressive activity. Cell Adh Migr 1: 196-198, 2007.

14. Muranen T, Grönholm M, Lampin A, et al: The tumor suppressor merlin interacts with microtubules and modulates Schwann cell microtubule cytoskeleton. Hum Mol Genet 16: 1742-1751, 2007

15. Kissil JL, Wilker EW, Johnson KC, et al: Merlin, the product of the Nf2 tumor suppressor gene, is an inhibitor of the p21-activated kinase, Pak1. Mol Cell 12: 841-849, 2003.

16. Alfthan K, Heiska L, Grönholm M, et al: Cyclic AMP-dependent protein kinase phosphorylates merlin at serine 518 independently of p21-activated kinase and promotes merlin-ezrin heterodimerization. J Biol Chem 279: 18559-18566, 2004.

17. Jin H, Sperka T, Herrlich P and Morrison H: Tumorigenic transformation by CPI-17 through inhibition of a merlin phosphatase. Nature 442: 576-579, 2006.

18. Bruce B, Khanna G, Ren L, et al: Expression of the cytoskeleton linker protein ezrin in human cancers. Clin Exp Metastasis 24: 69-78, 2007.

19. Hunter KW: Ezrin, a key component in tumor metastasis. Trends Mol Med 10: 201-204, 2004.

20. Yu Y, Khan J, Khanna C, et al: Expression profiling identifies the cytoskeletal organizer ezrin and the developmental homeoprotein Six 1 as keymetastatic regulators. Nat Med 10: 175-181, 2004.

21. Ohtani K, Sakamoto H, Rutherford T, et al: Ezrin, a membranecytoskeletal linking protein, is involved in the process of invasion of endometrial cancer cells. Cancer Lett 147: 31-38, 1999.

22. Hiscox S and Jiang WG: Ezrin regulates cell-cell and cell-matrix adhesion, a possible role with E-cadherin/beta-catenin. Cell Sci 112: 3081-3090, 1999.

23. Ren L, Hong SH, Cassavaugh J, et al: The actin-cytoskeleton linker protein ezrin is regulated during osteosarcoma metastasis by PKC. Oncogene 28: 792-802, 2009.

24. Wittekind C and Oberschmid B: TNM classification of malignant tumors 2010: General aspects and amendments in the general section. Pathologe 31: 333-338, 2010 (In German).

25. Cui Y, Li T, Zhang D and Han J: Expression of Ezrin and phosphorylated Ezrin (pEzrin) in pancreatic ductal adenocarcinoma. Cancer Invest 28: 242-247, 2010.

26. Mathew J, Hines JE, Obafunwa JO, et al: CD44 is expressed in hepatocellular carcinomas showing vascular invasion. J Pathol 179: 74-79, 1996.

27. Moilanen J, Lassus H, Leminen A, et al: Ezrin immumoreactivity in relation to survival in serous ovarian carcinoma patients. Gynecol Oncol 90: 273-281, 2003. 